\title{
Manipulation of response-contingent unconditioned-stimulus intensity in human eyelid conditioning: A two-phase model analysis
}

\author{
CRAIG G. CLARK and WILLIAM F. PROKASY \\ University of Utah, Salt Lake City, Utah 84112
}

\begin{abstract}
Four groups of human subjects were given 360 classical eyeblink conditioning trials. All groups received the same UCS (unconditioned stimulus) intensity on $\overline{C R}$ (nonconditioned response) trials but differed in the intensity presented on CR trials. Response probability increased as a positive function of UCS intensity on CR trials. Phase 1 of the two-phase model was longer when no UCS was presented on CR trials, but did not differ in duration among the remaining three groups. Most subjects could be described with a single operator in Phase 2, the operator limit increasing as a positive function of CR-contingent UCS intensity. For subjects requiring different operator limits on $\mathrm{CR}$ and $\overline{\mathrm{CR}}$ trials, the latter was lower with high $\mathrm{CR}$-trial intensities but higher with low CR-trial intensities. The results were interpreted to be more consistent with drive theory than with "law-of-effect" or two-factor theories.
\end{abstract}

Coleman (1972) and Gormezano and Coleman (1973) have recently provided an empirical test of "law-of-effect" accounts of classical conditioning performance. Such accounts (see, e.g., Hebb, 1956; Perkins, 1955, 1968) have held that the source of reinforcement in classical defense conditioning arises from $C R$ (conditioned response) attenuation of the aversive UCS (unconditioned stimulus). To examine this contention. Coleman (1972) manipulated CR-contingent UCS intensity in a classical aversive conditioning situation with rabbits as subjects and frequency of nictitating membrane responses as the dependent measure. They exposed different groups of subjects to different CR-contingent intensities while holding $\overline{\mathrm{CR}}$-contingent intensity constant. The principal result was inconsistent with theoretical expectations: percentage CRs decreased as the level of CR-contingent UCS intensity decreased. However, since the decrease in percentage CRs was also accompanied by decreases in the mean UCS intensity delivered to each group, the results were interpreted to be consistent with drive theory (e.g., Spence, 1956).

The purpose of the present study is to examine the effects of CR-contingent UCS intensity on human subjects with the relative frequency of the conditioned eyelid reflex as the dependent measure. Because it has been successful in describing aversive conditioning. the two-phase model was employed for data analysis (Prokasy, 1972, 1973, 1974; Prokasy, Williams, Lee.

\footnotetext{
This paper was submitted by the junior author to the faculty of the University of Utah in partial fulfillment of the requirements of the degree of Master of Science. Research support was provided by NSF Grant MBS 71-01219 to William F. Prokasy. The authors would like to thank William C. Williams and William Y. M. Lee for their assistance in computer programming. The authors would also like to thank John R. Dietlein and Howard K. Lee for aid in testing subjects.
}

\& Kumpfer, 1974). The model has two phases. During Phase 1 , a subject responds at a constant rate $\left(\mathrm{P}_{\mathrm{O}}\right)$, and during Phase 2 , increments and decrements can occur depending upon the outcome of the previous trial. $\mathrm{P}_{i}$, response probability on Trial $i$, is expressed as:

(1) $\mathrm{P}_{\mathrm{i}}=\mathrm{P}_{\mathrm{o}}, \mathrm{i}=1, \mathrm{~K}$,

(2) $P_{i}=P_{i-1}+\Theta_{1}\left(\lambda_{1}-P_{i-1}\right), i>K$, given $C R$ on trial $\mathrm{i}-1$,

(3) $\mathrm{P}_{\mathbf{i}}=\mathrm{P}_{\mathbf{i}-1}+\Theta_{2}\left(\lambda_{2}-\mathrm{P}_{\mathbf{i}-1}\right), \mathrm{i}>\mathrm{K}$, given $\overline{\mathrm{CR}}$ on trial i - 1 ,

where $K+1$ is the trial on which a subject enters Phase 2, $\Theta_{1}$ and $\Theta_{2}$ are growth parameters, and $\lambda_{1}$ and $\lambda_{2}$ are asymptotes. It will be noted that during Phase 2 one operator applies to CR trials and the other to $\overline{\mathrm{CR}}$ trials.

In addition to providing a concise description of the effects of an independent variable, the two-phase model allows one to examine particular theories in a more precise way than is otherwise possible. Whereas theories of conditioning have typically been evaluated in terms of their predictions of overall performance measures, the present study provides an evaluation in terms of the more specific trial-by-trial changes in performance associated with the parameters of the two-phase model.

The aforementioned "law-of-effect" theorists (Hebb, 1956; Perkins, 1955, 1968) have maintained that the CR is reinforced by its modification of the stimulus consequences of the UCS. To the extent that these theorists would argue that this mechanism provides the sole source of reinforcement, the CS- $\overline{C R}-\mathrm{UCS}$ trial would not be expected to alter CR probability, and thus $\theta_{2}$ should equal 0.0 . Since decreases in the level of CR-contingent UCS intensity should improve the stimulus consequences of a $C R$, one might expect corresponding increases in $\Theta_{1}, \lambda_{1}$, or both. Perkins 
(1968), however, might argue that a subject compares the relative attractiveness of $C R$ and $\overline{C R}$ trials, prefers the former, and moves toward a common limit (i.e., $\lambda_{1}$ $=\lambda_{2}$ ) following both trials. According to this interpretation, one would expect decreases in the level of CR-contingent UCS intensity to increase the value of the common limit. The $\theta$ s need not be equal, but both should exceed 0.0 .

Considerably different predictions arise from drive theory according to Spence $(1956,1960, \mathrm{pp} .109-110)$ and Spence, Haggard, and Ross (1958). Drive is a function of the overall average intensity of the UCS, while the increment of habit strength on a particular trial is a function of the intensity of the UCS on that trial. Because $C R$ presence-absence is not an important variable in itself, the same UCS intensity on CS-CR-UCS and CS-CR-UCS trials should result in a single habit strength limit. Consider, now, a situation in which the intensity of the UCS is different on CS-CR-UCS and CS-CR-UCS trials. Since habit is a function of UCS intensity, and since, as provisionally implied in the theory, habit strength does not decrease, a larger value of $\theta$ should be associated with the higher UCS intensity.

The avoidance situation represents a special case in Spencian theory. In the absence of a UCS, habit strength is not acquired, but inhibition is. Therefore, $\lambda_{1}$ should be less than $\lambda_{2}$, and $\lambda_{1}$ should be very low. It is to be noted from a different theoretical perspective that the proposition that avoidance subjects receive the UCS when "needed" also implies $\lambda_{1}<\lambda_{2}$ (see, e.g., Gormezano \& Coleman, 1973, p. 54; Moore \& Gormezano, 1961, pp. 557-558; Runquist, Sidowski, \& Gormezano, 1962, p. 49).

Two additional predictions may be made from Spence's theory. As a consequence of increased drive, operator limits should increase with increases in mean UCS intensity. Since increases in the limit of reaction potential decrease the number of trials required to reach threshold, $K$ should decrease with increases in mean UCS intensity.

Two-factor theory (see, e.g., the summary by Rescorla \& Solomon, 1967) assumes that a CS acquires motivating properties (e.g., fear, anxiety, pain. etc.) when paired with an aversive UCS. Thus, the CS-CR-UCS trial, through an increase in motivating properties of the CS, should result in an increase in response probability. The CS-CR-DCS trial, however, should have two consequences: CR reinforcement due to fear reduction, and a reduction in the motivating properties of the CS. The former should increase response strength, while the latter should decrease response strength. In order to account for the extinction of avoidance responses, the theory must assume that, at some point in training, the net impact of these two effects on response strength is a decrement in response probability. Thus, two-factor theory makes the identical prediction concerning avoidance behavior made by drive and "need" interpretations: $\lambda_{1}<\lambda_{2}$.

\section{METHOD}

\section{Apparatus}

Eyelid movements were converted to voltage changes by means of a wire attached to the subject's right eyelid and to the shaft of a microtorque potentiometer (see Prokasy \& Whaley, 1961). Responses were amplified and recorded by a Beckman Type $\mathbf{R}$ dynograph, using a 482AM8 power amplifier and a paper speed of $50 \mathrm{~mm} / \mathrm{sec}$. The $482 \mathrm{AM} 8$ amplifier provided a $\pm 1 \mathrm{~V}$ dc RMS output to the A/D converter of a DEC PDP-12/A computer. The paper record provided a check against the computer-scored record.

Stibjects were seated in one of two separate $1.8 \times 1.8 \mathrm{~m}$ cubicles. A stimulus panel, consisting of lights behind an $8 \times 55 \mathrm{~cm}$ strip of plastic. was located approximately $1 \mathrm{~m}$ in front of subjects at eye level.

The CS (conditioned stimulus) was a light rated at $2 \mathrm{~W}$, illuminated for $501 \mathrm{msec}$. The UCS was a $50-\mathrm{msec}$ puff of nitrogen delivered to the cornea of the subject's right eye at CS offset. Three levels of UCS intensity were employed: sufficient pressure to support a $50-, 150-$, or $250-\mathrm{mm}$ column of mercury.

The PDP-12/A computer programmed the ISIs (interstimulus intervals), ITIs (intertrial intervals), CSs, and UCSs. The mean ITI was $7 \mathrm{sec}$, with $6-, 7-$, and 8-sec ITIs sequenced unsystematically. The ISI was $516 \mathrm{msec}$. This value was determined by switching the UCS on $501 \mathrm{msec}$ after CS onset and measuring the total lag $(15 \mathrm{msec})$ between the UCS onset pulse and UCS impact on the cornea. The solenoid delay was approximately $12 \mathrm{msec}$, and line delivery lag was approximately $3 \mathrm{msec}$.

\section{Subjects}

The subjects were 175 male and female student volunteers from introductory psychology classes at the University of Utah. Nine students were lost due to apparatus failure or experimenter error. Fifteen students were deleted because they began more than 10 consecutive trials with eyes closed.

\section{CR Definition}

CRs were scored by the computer, employing amplitude and slope criteria. Voltage changes across potentiometers were sampled by the analog channels once every $3 \mathrm{msec}$. The program was calibrated so that a voltage change equivalent to a $1-\mathrm{mm}$ deflection of the galvanometer pen defined the amplitude criterion. If the amplitude criterion was met within three consecutive samples (6 msec), a response was registered. Only those responses occurring from $150 \mathrm{msec}$ after CS onset until CS offset were counted. Forty protocols were examined for discrepancies between hand and machine scoring. Of a total of 16,000 trials, on only $21(.131 \%)$ was there a discrepancy. Of these, 15 were erroneous detections and 6 were erroneous omissions.

\section{Experimental Dedign}

A classical cond itioning group (Group $C L, N=37$ ) received the $150 \cdot \mathrm{mm}$ UCS on all trials. An avoidance conditioning group (Group AV, $N=38$ ) received the $150-\mathrm{mm}$ UCS on $\overline{C R}$ trials and no UCS on CR trials. A response-contingent decremental group (Group DE, $N=38$ ) received the $150-\mathrm{mm}$ UCS on $\overline{\mathrm{CR}}$ trials and the $50 . \mathrm{mm}$ UCS on CR trials. A response-contingent incremental group (Group IN, $N=38$ ) received the $150-\mathrm{mm}$ UCS on $\overline{\mathrm{CR}}$ trials and the $250-\mathrm{mm}$ UCS on CR trials.

\section{Procedure}

Subjects were assigned randomly to groups and tested in parallel whenever possible. Brief tape-recorded instructions explained the stimuli to be employed and requested that the subjects sit quietly and let their reactions take care of themselves. All subjects received the same sequence of $\mathbf{4 0}$ adaptation trials, consisting of a random 
sequence of $30 \mathrm{CSs}$ and 10 UCSs, at the $150-\mathrm{mm}$ intensity. Adaptation trials were followed by 360 conditioning trials. Each experimental session lasted $53 \mathrm{~min}$. Following each session subjects completed written questionnaires designed to determine whether or not they detected differences in UCS intensities and whether or not they detected response-contingent differences in UCS intensities.

\section{RESULTS}

Mean response probability over the last 100 conditioning trials decreased with decreases in the intensity of the CR-contingent UCS, $F(3,147)=8.03$, $M S_{e}=.042(p \leqslant .05$ was the rejection region in this and subsequent statistical rests). Mean response probability over these trials was $.813, .799, .752$, and .606 in Groups IN. CL, DE, and AV, respectively. Note that the ordering of the means is precisely in the direction of the intensity of the UCS on CR trials.

\section{Parameter Estimates}

The details of the parameter estimation and subject categorization procedures have been described elsewhere (Prokasy, 1973). Briefly, the computer subroutine STEPIT (Chandler, 1969) was used to estimate maximally likely parameters for each subject under four different Phase 2 assumptions:
(A) $\theta_{1}=\theta_{2}, \lambda_{1}=\lambda_{2}$
(B) $\Theta_{1} \neq \Theta_{2}, \lambda_{1}=\lambda_{2}$,
(C) $\Theta_{1}=\theta_{2}, \lambda_{1} \neq \lambda_{2}$
(D) $\theta_{1} \neq \theta_{2}, \lambda_{1} \neq \lambda_{2}$.

Assumption $A$ is the simplest in that each trial contributes to subsequent response probability according to the same linear operator. Assumptions $B, C$, and $D$, however, are more complex in that different linear operators correspond to $\mathrm{CR}$ and $\overline{\mathrm{CR}}$ trials. Since the model implied by Assumption A is a submodel of those implied by Assumptions B and C, and since the latter two are submodels of that implied by Assumption D, a maximum-likelihood ratio test may be used to determine which assumption is sufficient for each subject's protocol (for further details, see Prokasy, 1973; Theios, 1968).

The subroutine STEPIT yields parameter estimates at a relative maximum. With the present class of models. such estimates may, on occasion, poorly approximate those at the absolute maximum. To avoid this problem, starting points for STEPIT were drawn from a table of random numbers and three separate sets of estimates were made. By this procedure, the likelihood of failing to obtain parameter estimates which closely approximate those at the absolute maximum is less than .02 .

\section{Manipulation of CR-Contingent UCS Intensity}

The number of subjects categorized according to each assumption, with the corresponding mean parameter values, are provided in Table 1 . For most
Table 1

Mean Parameter Estimates for Assumption Categories Within Each Group

\begin{tabular}{|c|c|c|c|c|c|c|}
\hline Group & $\begin{array}{l}\text { ssumption } \\
\text { Category }\end{array}$ & $\mathbf{N}$ & $\theta_{1}$ & $\lambda_{1}$ & $\theta_{2}$ & $\lambda_{2}$ \\
\hline $\begin{array}{l}\text { IN } \\
\left(\mathrm{P}_{\mathrm{O}}=.132\right) \\
(\mathrm{K}=12.6)\end{array}$ & $\begin{array}{l}\text { A } \\
B \\
C \\
D\end{array}$ & $\begin{array}{r}30 \\
1 \\
5 \\
2\end{array}$ & $\begin{array}{l}.374 \\
.000 \\
.432 \\
.128\end{array}$ & $\begin{array}{l}.841 \\
\\
.721 \\
.467\end{array}$ & $\begin{array}{l}.374 \\
.141 \\
.432 \\
.590\end{array}$ & $\begin{array}{l}.841 \\
.930 \\
.380 \\
.791\end{array}$ \\
\hline $\begin{array}{l}\mathrm{CL} \\
\left(\mathrm{P}_{\mathrm{o}}=.142\right) \\
(\mathrm{K}=12.5)\end{array}$ & $\begin{array}{l}A \\
B \\
C \\
D\end{array}$ & $\begin{array}{r}25 \\
3 \\
4 \\
5\end{array}$ & $\begin{array}{l}.538 \\
.000 \\
.246 \\
.240\end{array}$ & $\begin{array}{l}.788 \\
\\
.791 \\
.543\end{array}$ & $\begin{array}{l}.538 \\
.338 \\
.246 \\
.417\end{array}$ & $\begin{array}{l}.788 \\
.976 \\
.409 \\
.764\end{array}$ \\
\hline $\begin{array}{l}\mathrm{DE} \\
\left(\mathrm{P}_{\mathrm{o}}=.118\right) \\
(\mathrm{K}=12.2)\end{array}$ & $\begin{array}{l}\mathrm{A} \\
\mathrm{B} \\
\mathrm{C} \\
\mathrm{D}\end{array}$ & $\begin{array}{r}34 \\
2 \\
1 \\
1\end{array}$ & $\begin{array}{r}.416 \\
.000 \\
1.000 \\
.001\end{array}$ & $\begin{array}{l}.755 \\
\\
.844 \\
.000\end{array}$ & $\begin{array}{r}.416 \\
.206 \\
1.000 \\
.749\end{array}$ & $\begin{array}{r}.755 \\
.920 \\
.941 \\
1.000\end{array}$ \\
\hline $\begin{array}{l}\mathrm{AV} \\
\left(\mathrm{P}_{\mathrm{O}}=.166\right) \\
(\mathrm{K}=26.2)\end{array}$ & $\begin{array}{l}\text { A } \\
\text { B } \\
\text { C } \\
\text { D }\end{array}$ & $\begin{array}{r}21 \\
2 \\
14 \\
1\end{array}$ & $\begin{array}{l}.340 \\
.000 \\
.379 \\
.001\end{array}$ & $\begin{array}{l}.620 \\
\\
.513 \\
.000\end{array}$ & $\begin{array}{l}.340 \\
.290 \\
.379 \\
.383\end{array}$ & $\begin{array}{r}.620 \\
.907 \\
.567 \\
1.000\end{array}$ \\
\hline
\end{tabular}

Note-Values of $P_{0}$ and $K$ were averaged across all subjects in each group.

subjects (110), Assumption A was sufficient. Fewer subjects (24) required Assumption C, and fewer still required Assumptions D and B ( 9 and 8, respectively).

Simulation procedures were employed to determine the power of the maximum-likelihood ratio test in rejecting the null hypothesis since it could not be assumed that maximum-likelihood ratios obtained with the present class of models would be distributed exactly as chi square. The ratios employed in this study yielded the following simulation results: if Assumption A were true, the probability of classifying a subject as meeting Assumptions B, C, or D would be .11 ; if Assumption $C$ were true, the probability of classifying a subject as meeting Assumption A or Assumption B would be .24. Simulation also shows that with $\Theta$ values of .1 and above, and with subjects having but a single limit, there is not enough information in the data to permit reliable within-subject differentiation of $\theta_{s}$ even if Assumption B were true. Thus, it cannot be assumed that the failure to categorize subjects under Assumption $B$ means that $\Theta$ s are equal on $C R$ and $\overline{C R}$ trials. Fortunately, this is not critical for present theoretical purposes.

One source of bias should be noted. Under Assumption A, with values of $\theta$ in the vicinity of .1 and with values of $\lambda$ within .2 of $P_{0}$, both $K$ and $\Theta$ tend to be overestimated. In the present study, for only seven subjects were estimates of $\lambda$ within .2 of $P_{0}$ and either $K$ or $\Theta$ greater than the average for their group. The impact of this bias is apparently trivial for present purposes. Having noted this source of bias, it should be emphasized that, otherwise, estimates of $K$, 
$\Theta$. and $\lambda$ were, in our simulations, very close to theoretical values.

As suggested by the table, the number of subjects requiring Assumption $\mathrm{C}$ was a $\mathrm{U}$-shaped function of the level of CR-contingent UCS intensity, $\chi^{2}(3)=$ 18.38. The greatest number of Assumption $C$ subjects was required by Group AV, the smallest number by Group DE, and intermediate numbers by Groups $\mathrm{CL}$ and IN.

The independent variable affected operator limits in two ways. First. for those subjects who required but a single $\lambda$ (i.e., Assumption A and Assumption B subjects), mean estimates of $\lambda$ increased with increases in CR-contingent intensity, $F(3,144)=$ $6.48, \mathrm{MS}_{\mathrm{e}}=.040$. As with mean response probability at the end of training, mean estimates of $\lambda$ were ordered precisely in the direction of the intensity of the UCS on CR trials. Second, it appears that whether or not $\lambda_{1}$ is less than $\lambda_{2}$ depends upon the intensity of the CR-contingent UCS. For example, the proportion of subjects for whom $\lambda_{1}<\lambda_{2}$ in Groups $\mathrm{AV}$ and $\mathrm{DE}$ was .69 , while the corresponding proportion in Groups $\mathrm{CL}$ and IN was .25. The likelihood of a proportion discrepancy that large or larger is .016 by Fisher's exact-probability test.

There were no effects of contingent intensity on $\Theta$. It is to be noted, however, that for no subject did $\Theta_{2}=$ 0.0 . For 16 of the 17 subjects requiring two $\Theta$ s (i.e., Assumption B and Assumption D subjects), $\theta_{1}$ was less than $\Theta_{2}, \chi^{2}(1)=13.24$. Differential $\Theta$ s, when both are greater than zero, should be interpreted with some caution. The likelihood, based on our simulations, of categorizing an Assumption A subject under Assumption B is about .002. The sizable percentage of subjects $(11 \%)$ requiring a differentiation between $\Theta_{1}$ and $\theta_{2}$ does, however, suggest that the effect is genuine. For the eight Assumption B subjects, $\Theta_{1}=0.0$.

There were also no effects of the independent variable on group distributions of $\mathbf{K}$; for each group, the distribution of $\mathrm{K}$ was positively skewed with a mode of three.

\section{Classical vs. Avoidance}

Because comparisons of classical and avoidance conditioning preparations have been of particular interest in conditioning research, separate analyses contrasting Groups $\mathrm{CL}$ and $\mathrm{AV}$ were made. First, the average estimate of the common $\lambda$ (i.e., pooled across Assumptions A and B) was greater in Group CL than in Group AV, $t(49)=3.10$. In addition, there was a greater proportion of Assumption $C$ subjects in Group AV (.64) than in Group CL (.00) with $\lambda_{1}<\lambda_{2}$, Fisher's exact probability $=.041$. While the mean $\mathrm{K}$ was greater in Group AV than in Group CL, this difference was not reliable by a $t$ test. That this was not reliable is apparently due to the skewed distributions of $\mathrm{K}$, since Group AV did not have a reliably greater proportion of subjects (.45 vs. .19) with $K$ values greater than nine, $\chi^{2}(1)=5.74$.

\section{Questionnaire Data}

Questionnaires were analyzed to determine whether or not subjects detected changes in UCS intensity. A liberal criterion simply required subjects to make any reference either to the omission of the UCS or to changes in its intensity. Subjects did not meet criterion if they indicated that intensity changes were due to their adaptation to the UCS. The number of subjects in Groups AV, DE, CL, and IN who met the detection criterion were $37,35,20$, and 26 , respectively. Of particular interest is the finding that the proportion of subjects reporting intensity changes did not differ reliably between Groups $C L$ and IN, $\chi^{2}(1)=1.61$. However, Groups AV and DE did differ reliably from Group CL in this regard, $\chi^{2}(1)=16.98$ and 13.88, respectively. Only a very few subjects indicated that such intensity changes were response contingent: $11,7,2$, and 3 in Groups AV, DE, CL, and IN, respectively.

\section{DISCUSSION}

In general, the results of manipulations of CR-contingent UCS intensity in human eyelid conditioning were consistent with those involving rabbit nictitating membrane conditioning (Coleman, 1972): that is, performance measures increased with increases in the intensity of the CR-contingent UCS. For this reason, the conclusions of Gormezano and Coleman (1973) were, for the most part, confirmed.

Three "law-of-effect" interpretations of classical defense conditioning may be examined: (a) that reinforcement is the exclusive consequence of events coincident with the CR, (b) that CS-UCS contiguity accounts for initial acquisition, while $C R$ reinforcement predominates later in training, and (c) that CR presence is relatively more reinforcing than $C R$ absence. The first, a strict reinforcement position (see, e.g., Hebb, 1956), cannot be sustained in light of the fact that $\overline{C R}$ trials did contribute to performance $\left(\Theta_{2}>0.0\right)$.

The second position, by emphasizing the role of $C R$ reinforcement (see. e.g., Birch \& Bitterman, 1949; Jones, 1962; Maier \& Schneirla, 1942), implies a special relationship between operator limits. On CS- $\overline{C R}-U C S$ trials, contiguity is the only operative mechanism. However, on CS-CR-UCS trials both contiguity and reinforcement are operative and their combined effect would be expected to result in a higher limit (i.e., $\lambda_{1}>\lambda_{2}$ ). Higher limits should also be associated with CS-CR-DCS trials, since reinforcement alone should maintain higher rates than contiguity alone. While some support for this position derives from the fact that $\lambda_{1}$ was generally greater than $\lambda_{2}$ for a minority of subjects in Groups IN 
and CL, it is difficult to see how the sufficiency of a single limit for a majority of subjects in all groups may be reconciled with the theory considering the importance of CR reinforcement. Also contrary to this position is the fact that $\lambda_{1}$ was generally less than $\lambda_{2}$ when two limits were required in Groups DE and AV.

The third position, a relative reinforcement theory (see, e.g., Perkins, 1968), implies a single operator limit common to $\mathrm{CR}$ and $\overline{\mathrm{CR}}$ trials. This is because the comparison of the relative attractiveness of both $\mathrm{CR}$ and $\overline{\mathrm{CR}}$ trials provides the basis for what is reinforced. According to this view, one would expect increases in the common limit with decreases in the intensity of a CR-contingent aversive stimulus. The $\Theta \mathrm{s}$ need not be equal, but both should exceed 0.0 . Three outcomes are inconsistent with Perkins' theory: some subjects required two limits, $\Theta_{1}$ was equal to 0.0 for some subjects, and decreases in the average estimate of the common limit corresponded to decreases in the intensity of the CR-contingent UCS. It is difficult to see how the latter two outcomes may be reconciled with any "law-of-effect" interpretation of classical defense conditioning.

Consistent with the conclusions of Gormezano and Coleman (1973), drive theory (Spence, 1956, 1960) is supported at a general level by the present study. The ordering of the common limits, for example, is the predicted consequence of differing levels of drive, which is determined by the average UCS intensity. The insignificance of $C R$ presence-absence, a provisional implication of the theory, is indicated by the fact that a single operator was sufficient for most subjects in Groups IN, CL, and DE. This conclusion appears to be supported by the fact that when two limits were required in Groups IN and DE, the higher limit was generally associated with the greater UCS intensity. By allowing either habit strength or drive to modify within a session in which multiple UCSs are employed, this relationship between $\lambda_{1}$ and $\lambda_{2}$ would become coordinate with the theory. That the predicted growth rate differences were not observed in Group IN may be a consequence of the failure of subjects in that group to discriminate two UCS intensities. However, the finding that the number of two-operator subjects was suppressed in Group DE. where UCS intensities were discriminated, is inconsistent with the theory. In addition, the presence of two-operator subjects in Group CL, where only one intensity was employed, is also inconsistent with the theory. The failure to observe the predicted differences in mean $\mathrm{K}$ simply reveals the inability of the independent variable to affect the length of Phase 1 . This does not constitute evidence against the theory, since differences in mean $K$ have been observed previously as a result of manipulations of UCS intensity (Prokasy, 1973).

Drive theory implies $\lambda_{1}<\lambda_{2}$ for avoidance conditioning because it states that habit strength increases following CS- $\overline{\mathrm{CR}}$-UCS trials and that inhibition develops following CS-CR- $\overline{U C S}$ trials. The finding that $\lambda_{1}$ was generally less than $\lambda_{2}$ for two-operator subjects in Group AV is consistent with this prediction. That a single limit was sufficient for most subjects in this group presents some difficulty for the theory, although it should be noted that a growth function for inhibition has not been specified.

Two-factor theory also implies $\lambda_{1}<\lambda_{2}$. As pointed out earlier, the CS- $\overline{C R}$-UCS trial, by increasing the motivating properties of the CS, should result in an increase in response probability. The net impact of the CS-CR-UCS trial, at least late in training, should be a decrement in response probability. The theory is supported by the fact that $\lambda_{1}$ was generally less than $\lambda_{2}$ for those subjects requiring two limits in Group AV. Although the theory does not require that the net impact of the CS-CR-UCS trial be a decrement in response probability, it is not apparent what additional assumptions would be required to account for the one-operator subjects in that group. The five subjects for whom $\lambda_{1}$ was greater than $\lambda_{2}$ present more difficulty for the theory, since, for them, once $P_{i}$ exceeded $\lambda_{2}$, response decrements followed the CS- $\overline{C R}$-UCS trial even though it should result in an increase in the motivating properties of the CS. It should be noted that this relationship, $\lambda_{1}>\lambda_{2}$, was the outcome for discriminated avoidance leg flexions in dogs and cats (Prokasy, 1974), thus suggesting that two-factor theory does not fare well upon close examination of the data.

Some difficulties involved in using past avoidance data to support a theory merit attention. Consider the following common view: CS-CR-UCS trials lead to acquisition and CS-CR- $\overline{\mathrm{UCS}}$ trials lead to extinction (see, e.g.. Hull, 1929, p. 510; Schlosberg, 1937, pp. 380-381; Sheffield, 1948, pp. 174-175). Avoidance data in which runs of CS-CR-UCS trials have alternated with runs of CS-CR- $\overline{U C S}$ trials have been cited by the above authors in support of this position. It can be shown that such data contradict, rather than support, the above position. To illustrate, consider a subject with a stable mean response probability late in training of .80 . In terms of the two-phase model, three relationships are possible between $\lambda_{1}$ and $\lambda_{2}: \lambda_{1}=\lambda_{2}$, $\lambda_{1}>\lambda_{2}$, and $\lambda_{1}<\lambda_{2}$. If $\lambda_{1}=\lambda_{2}$, then the number of transitions between CRs and CRs is determined by a binomial distribution in which the probability of a CR on trial $i, p\left(C R_{\mathfrak{i}}\right)$, equals .80 , regardless of trial outcome. If $\lambda_{1}>\lambda_{2}$, and (for simplicity) $\theta_{1}=\theta_{2}=$ 1.0 , then $\mathrm{p}\left(\mathrm{CR}_{\mathrm{i}} / \mathrm{CR}_{\mathrm{i}-1}\right)>.80$ and $\mathrm{p}\left(\mathrm{CR}_{\mathbf{i}} / \overline{\mathrm{CR}}_{\mathrm{i}-1}\right)<$ .80 . This, in turn, results in fewer transitions between CRs and $\overline{\mathrm{CR}} \mathrm{s}$ than would be expected if $\lambda_{1}=\lambda_{2}$. If, on the other hand, $\lambda_{1}<\lambda_{2}$, and (again for simplicity) $\theta_{1}$ $=\theta_{2}=1.0$, then $\mathrm{p}\left(\mathrm{CR}_{\mathrm{i}} / \mathrm{CR}_{\mathrm{i}-1}\right)<.80$ and $\mathrm{p}\left(\mathrm{CR}_{\mathrm{i}} / \overline{\mathrm{CR}}_{\mathrm{i}-1}\right)>.80$. This results in more transitions between CRs and $\overline{C R}$ than would be expected if $\lambda_{1}=$ $\lambda_{2}$. The important point is that this last relationship, 
$\lambda_{1}<\lambda_{2}$, is implied by the theory that CS- $\overline{\mathrm{CR}}$-UCS trials lead to increments and CS-CR-DCS trials lead to decrements. In contrast, data in which zuns of $\overline{C R} s$ alternated with runs of CRs reflects $\lambda_{1}>\lambda_{2}$. Thus, it would appear that past data cited in support of this theory actually contradict it.

\section{REFERENCES}

Birch, H. G.. \& Bitterman, M. E. Reinforcement and learning: The process of sensory integration. Psychological Review. 1949, 56, 292-308.

ChANDLER, J. P. STEPIT: Finds local minima of a smooth function of several parameters (CPA 312). Behavioral Science, 1969, 14. $81-82$.

Coleman, S, R. Effects upon the rabbit's nictitating membrane response of response-contingent changes in intensity of the UCS. Unpublished doctoral dissertation, University of Iowa, 1972.

Gormezano, I., \& Coleman, S. R. The law of effect and CR contingent modification of the UCS. Conditional Reflex, 1973, 8, 41-56.

HEBв, D. O. The distinction between "classical" and "instrumental." Canadian Joumal of Psychology, 1956, 10, 165-166.

Huls, C. L. A functional interpretation of the conditioned reflex. Psychological Review, 1929, 36, 498-511.

JONES, J. E. Contiguity and reinforcement in relation to CS-UCS intervals in classical aversive conditioning. Psychological Review, 1962, 69, 176-186.

MAIER, N. R., \& SChneirla, T. C. Mechanisms in conditioning. Psychological Review, 1942, 49, 117-134.

MoORE, J. W., \& Gormezano, 1. Yoked comparisons in instrumental and classical eyelid conditioning. Journal of Experimental Psychology, 1961, 62, 552-559.

Perkins, C. C., JR. The stimulus conditions which follow learned responses. Psychological Review, 1955, 62, 341-348.

Perkins, C. C., JR. An analysis of the concept of reinforcement. Psychological Review. 1968, 75, 155-172.

Prokasy, W. F. Developments with the two-phase model applied to human eyelid conditioning. In A. H. Black \& W. F. Prokasy (Eds.). Classical conditioning II. New York: Appleton-Century-Crofts. 1972. Pp. 119-147.
Prokasy, W. F. A two-phase model account of aversive classical conditioning performance in humans and rabbits. Learming and Motivation, 1973, 4. 247-258.

Prokasy, W. F. Discriminated avoidance vs classical conditioning: A two-phase model analysis. Animal Learning \& Behavior, 1974, 2, 257-261.

Prokasy, W. F., \& Whaley, F. L. The intertrial interval in classical conditioning. Journal of Experimental Psychology, $1961,62,560-564$.

Prokasy, W. F., Williams, W. C., Lee, W. Y. M., \& Kumpfer, K. L. Two-phase model analysis of the effects of interstimulus interval and masking task in human aversive classical conditioning. Memory \& Cognition, 1974, 2, 206-210.

Rescorla, R. A., \& Solomon, R. L. Two-process learning theory: Relationships between Pavlovian conditioning and instrumental learning. Psychological Review, 1967, 74, 151-182.

Runquist, W. N., Sidowski, J., \& Gormezano, I. Yoked comparisons of classical and avoidance conditioning in differential conditioning of the eyelid response. Psychological Reports, 1962, 11, 43-50.

Schlosberg, $H$. The relationship between success and the laws of conditioning. Psychological Review, 1937, 44, 379-394.

SHEFFIELD, F. D. Avoidance training and the contiguity principle. Joumal of Comparative and Physiological Psychology, 1948, 58, 362-367.

SPENCE, K. W. Behavior theory and conditioning. New Haven: Yale University Press, 1956.

SPENCE, K. W. The roles of reinforcement and non-reinforcement in simple learning. In K. W. Spence (Ed.), Behavior theory and learning. Englewood Cliffs, N.J: Prentice-Hall, 1960.

SPENCE, K. W., Hagcard, D. F., \& Ross, L. F. UCS intensity and the associative (habit) strength of the eyelid CR. Journal of Experimental Psychology, 1958, 55, 404-411.

Theios, J. Finite integer models for learning in individual subjects. Psychological Review, 1968, 75, 292-307.

(Received for publication November 1, 1974; revision accepted August 27.1975 .) 